\title{
THE NEXT LINEAR COLLIDER DAMPING RING LATTICES*
}

\author{
A. Wolski ${ }^{\dagger}$, J.N. Corlett, LBNL, Berkeley, CA 94720, USA
}

\begin{abstract}
We report on the lattice design of the Next Linear Collider (NLC) damping rings. The damping rings are required to provide low emittance electron and positron bunch trains to the NLC linacs, at a rate of $120 \mathrm{~Hz}$. We present an optical design, based on a theoretical minimum emittance (TME) lattice, to produce the required normalized extracted beam emittances $\gamma \varepsilon_{\mathrm{x}}=3 \mathrm{~mm}$-mrad and $\gamma \varepsilon_{\mathrm{y}}=0.02 \mathrm{~mm}$ mrad. An assessment of dynamic aperture and non-linear effects is given. The positron predamping ring, required to reduce the emittance of the positron beam such that it may be accepted by a main damping ring, is also described.
\end{abstract}

\section{DAMPING REQUIREMENTS}

The main damping rings (MDRs) for the NLC are designed to accept a beam with normalized emittances both horizontally and vertically of $150 \mathrm{~mm}$-mrad. To achieve the specified luminosity, the transverse energy of the beam must be damped to normalized emittances of 3 $\mathrm{mm}$-mrad horizontally, and $0.02 \mathrm{~mm}$-mrad vertically. The positron source produces a beam with much larger emittances, up to $30,000 \mathrm{~mm}$-mrad. The necessary aperture of a damping ring that will accept this beam, is not compatible with the required extracted emittances and damping rate. We therefore need a pre-damping ring (PDR), to reduce the emittance of the positron beam to a value comparable with the beam from the electron source. The parameters driving the design of the damping rings are shown in Table 1. More details on the damping ring complex and subsystems are given in references [1],[2].

Table 1: Parameters Driving Damping Ring Design

\begin{tabular}{|l|l|}
\hline Beam energy & $1.98 \mathrm{GeV}$ \\
\hline Collider rep rate & $120 \mathrm{~Hz}$ \\
\hline Bunches per train & 190 \\
\hline Bunch separation & $1.4 \mathrm{~ns}$ \\
\hline Particles per bunch & $0.75 \times 10^{10}$ \\
\hline $\mathrm{e}^{-}$beam injected emittance (rms) & $150 \mathrm{~mm}-\mathrm{mrad}$ \\
\hline $\mathrm{e}^{+}$beam injected emittance (edge) & $30000 \mathrm{~mm}-\mathrm{mrad}$ \\
\hline Kicker rise/fall time (MDR/PDR) & $65 / 100 \mathrm{~ns}$ \\
\hline Extracted horizontal emittance $(\mathrm{rms})$ & $3.0 \mathrm{~mm}-\mathrm{mrad}$ \\
\hline Extracted vertical emittance $(\mathrm{rms})$ & $0.02 \mathrm{~mm}-\mathrm{mrad}$ \\
\hline
\end{tabular}

The parameters given in Table 1 , and other considerations, constrain the lattice designs. In particular, the MDR lattice should have:

- low natural emittance;

\footnotetext{
*Work supported by the US DOE under contract DE-AC03-76SF00098 †awolski@lbl.gov
}

- low vertical/horizontal emittance ratio;

- fast transverse damping times;

- good dynamic aperture, for injection efficiency.

\section{MAIN DAMPING RING LATTICE}

\subsection{Parameters}

Raubenheimer and Emma [3] have given an analysis of the relationships between the various lattice parameters for linear collider damping rings, leading to an optimal outline design. We applied the results of this analysis to the NLC, as a starting point for the detailed lattice design.

Table 2: Principal Parameters of the Main Damping Rings

\begin{tabular}{|l|l|l|}
\hline Energy & $E$ & $1.98 \mathrm{GeV}$ \\
\hline Circumference & $C$ & $299.792 \mathrm{~m}$ \\
\hline Number of stored trains & & 3 \\
\hline Natural emittance & $\gamma \varepsilon_{0}$ & $2.17 \mathrm{~mm}-\mathrm{mrad}$ \\
\hline Tunes & ${v_{\mathrm{x}}, \mathrm{v}_{\mathrm{y}}}$ & $27.2616,11.1357$ \\
\hline Natural chromaticity & $\xi_{\mathrm{x}}, \xi_{\mathrm{y}}$ & $-37.12,-28.24$ \\
\hline Momentum compaction & $\alpha$ & $2.95 \times 10^{-4}$ \\
\hline RF voltage & $V_{\mathrm{RF}}$ & $1.07 \mathrm{MV}$ \\
\hline RF acceptance & $\varepsilon_{\mathrm{RF}}$ & $1.5 \%$ \\
\hline Energy spread (rms) & $\sigma_{\delta}$ & $0.091 \%$ \\
\hline Bunch length (rms) & $\sigma_{\mathrm{z}}$ & $3.60 \mathrm{~mm}$ \\
\hline Integrated wiggler field & $\int_{B_{\mathrm{w}}}{ }^{2} \mathrm{ds}$ & $106.9 \mathrm{~T}^{2} \mathrm{~m}$ \\
\hline Energy loss/turn & $U_{0}+U_{\mathrm{w}}$ & $247+530 \mathrm{keV}$ \\
\hline Damping times & $\tau_{\mathrm{x}, \mathrm{y}, \varepsilon}$ & $4.85,5.09,2.61 \mathrm{~ms}$ \\
\hline
\end{tabular}

The principal parameters of the main damping rings are given in Table 2. The lattice is a racetrack design, with a total of 34 theoretical minimum emittance (TME) arc cells (plus four half-cells for matching into the $60 \mathrm{~m}$ long straight sections). The number of arc cells allows some detuning from the actual minimum emittance condition to improve the dynamics. The length of each cell is fixed by the need to keep the circumference, and hence the damping time, as low as possible, while allowing enough space in the straight sections for the injection/extraction systems on one side, and the damping wiggler on the other. The minimum circumference is determined by the actual length of each bunch train, and the rise and fall time of the injection and extraction kickers.

To achieve the required damping just from the dipole radiation would require unrealistically large dipole fields. In the current design, the main arc dipoles have a main field of $1.2 \mathrm{~T}$, and a vertically focusing gradient of 6.6 $\mathrm{Tm}^{-1}$. A wiggler provides most of the damping; the current design uses a hybrid wiggler of peak field $2.15 \mathrm{~T}$, period $0.27 \mathrm{~m}$ and total length $46 \mathrm{~m}$. 


\subsection{Linear Lattice Functions}

The beta functions and horizontal dispersion in one full arc cell are shown in Figure 1.

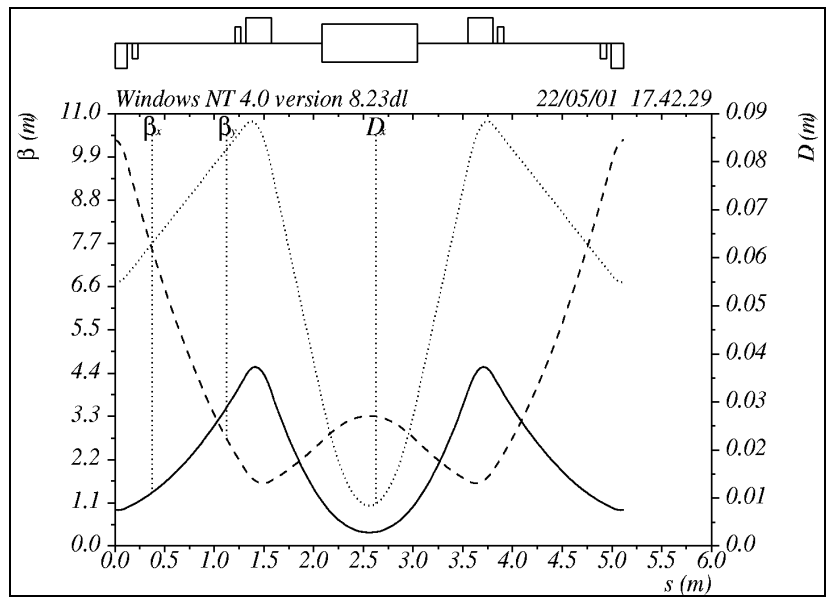

Figure 1: Lattice functions in one arc cell

The lattice functions in the injection/extraction straight are shown in Figure 2 where (in order of position along the straight) the circumference correction chicane, RF cavities, extraction kicker and septum, and injection septum and kicker can also be seen. The lattice in both straight sections is based on a simple FODO structure. In the wiggler straight, the wiggler itself provides the vertical focusing.

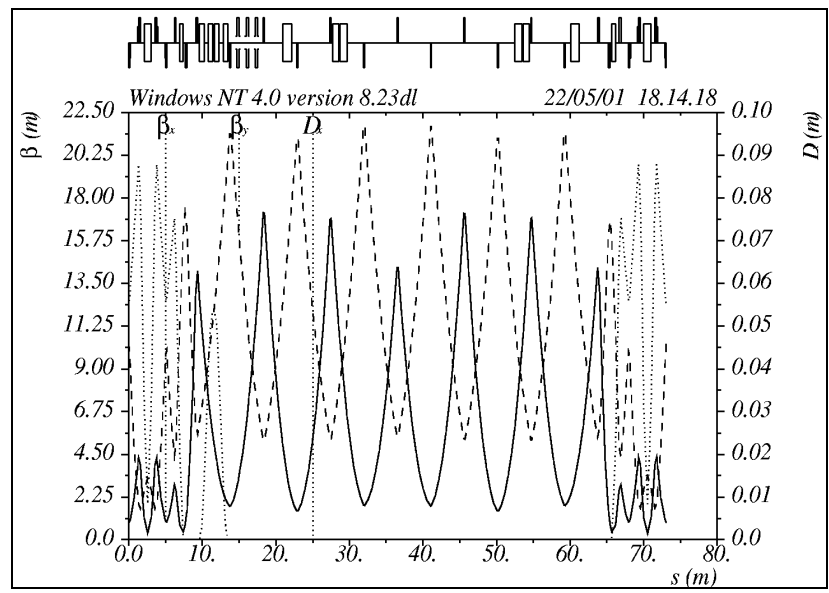

Figure 2: Injection/extraction straight lattice functions

The three RF cavities [4] are located before the extraction components, so that there are no transient changes in the loading on the cavities during an injection/extraction cycle. The RF frequency is $714 \mathrm{MHz}$, so that within a bunch train, every RF bucket is filled to give a bunch separation of $1.4 \mathrm{~ns}$. The RF frequency is locked to other systems within the NLC, so small corrections to minimize the energy error must be made by means of a chicane [5]. Experience at the ATF suggests that an adjustment range of $\pm 2 \mathrm{~mm}$ may be necessary.

The extraction kicker is separated from the injection kicker by a half-integer horizontal phase advance. This allows correction of any residual field seen by stored bunch trains as the kickers are turned on and off.

Figure 3 shows the working point of the lattice in tune space, with momentum deviations up to $\pm 2 \%$.

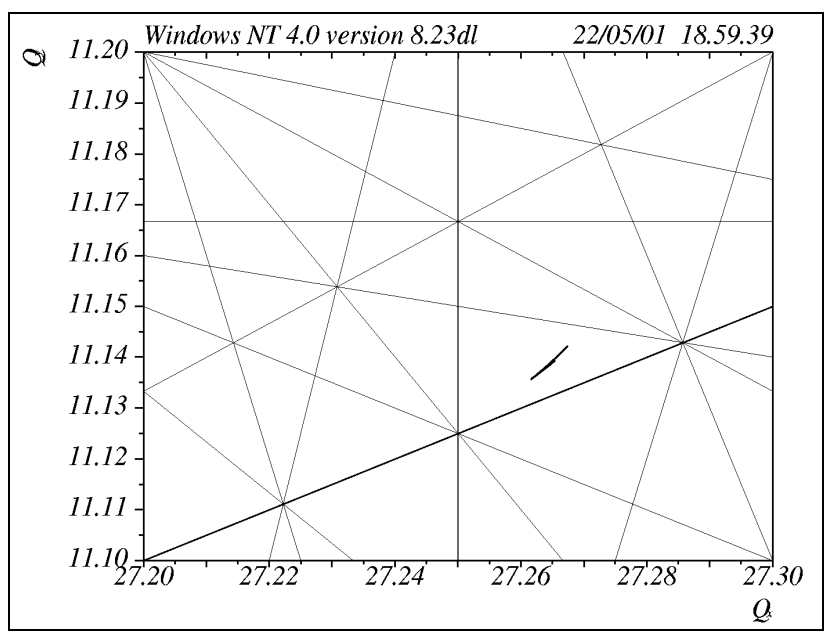

Figure 3: Working point in tune space

\subsection{Non-linear Dynamics}

A good dynamic aperture is required to ensure good injection efficiency, and thus avoid heavy radiation loads. Detailed calculations of the necessary aperture have not so far been carried out, but experience with the SLC damping rings suggests that the dynamic aperture should be at least 15 times the injected beam size. We initially optimized the dynamics of the lattice by working on a single arc cell. The phase advances over both straight sections were then adjusted to integer values; since all the components in these sections are (ideally) linear, the dynamics of the lattice are then essentially just the dynamics of a single cell. The non-linear components of the field in the damping wiggler do not significantly affect the dynamics, at least up to the physical aperture [6].

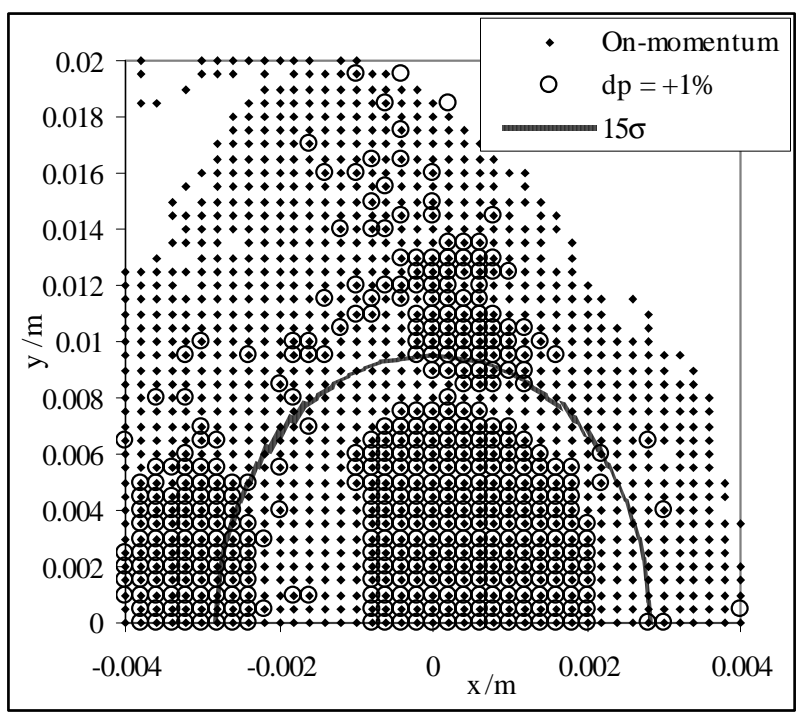

Figure 4: MDR dynamic aperture without errors 
Figure 4 shows the dynamic aperture of the bare lattice (no field errors or misalignments) with the wiggler modeled as a linear element. The dynamic momentum aperture of the lattice is estimated at $\pm 1 \%$. Tolerances on the field quality in the magnets have been determined by the criterion that the dynamic aperture should fall to no less than 15 times the injected beam size; the tolerances determined in this way appear reasonable.

\subsection{Orbit and Coupling Correction}

An equilibrium vertical emittance of $0.0118 \mathrm{~mm}-\mathrm{mrad}$ or lower is needed to achieve the $0.02 \mathrm{~mm}$-mrad extracted vertical emittance. Analytical estimates and simulations suggest that vertical dispersion and betatron coupling contribute about equally to the vertical emittance. Our correction scheme is still in development, but is currently based on two techniques:

- orbit correction with each quadrupole on a mover, and quadrupole-BPM offsets determined by beam based alignment;

- coupling correction with a skew quadrupole component superposed on each sextupole.

Simulations show that it may be possible to achieve the required vertical emittance just through orbit correction, but the BPM resolutions and alignment tolerances would be demanding. Use of skew quadrupoles at the sextupoles eases the tolerances considerably; the skew strengths are chosen to minimize the vertical dispersion. Since coupling in the sextupoles generates much of the vertical dispersion (after orbit correction), this technique effectively compensates the sextupole misalignments. The vertical dispersion must be measured with a precision better than $1 \mathrm{~mm}$; this can be achieved by averaging BPM readings. With the tolerances shown in Table 3, which are comparable to those achieved in the ALS, for example [7], our simulations show the required extracted emittance in more than $95 \%$ of cases.

Table 3: Tolerances for Vertical Emittance Control

\begin{tabular}{|l|l|}
\hline BPM resolution & $5 \mu \mathrm{m}$ \\
\hline BPM resolution with averaging & $0.3 \mu \mathrm{m}$ \\
\hline RMS BPM-Quadrupole alignment & $5 \mu \mathrm{m}$ \\
\hline Quadrupole mover step size & $1 \mu \mathrm{m}$ \\
\hline RMS sextupole alignment & $80 \mu \mathrm{m}$ \\
\hline
\end{tabular}

\subsection{Collective Limitations}

Studies of impedance effects from a variety of sources, including higher-order modes in the RF cavities, have been carried out and are reported elsewhere [8]. In general, beam quality will not be unduly limited, although a transverse feedback system will be required.

Studies have also been carried out of electron cloud effects and intra-beam scattering (IBS). An ante-chamber will minimize the density of photo-electrons in the positron ring; operation will be below the multipacting threshold, without a coating on the aluminum vacuum vessel to reduce secondary electron yield. Initial IBS calculations suggest that the vertical emittance increase could be $13 \%$. Studies are under way to verify IBS models in the MDR parameter regime [9], and more detailed and rigorous simulations are planned.

\section{PRE-DAMPING RING}

In the pre-damping ring, the main challenge is achieving the large acceptance. A basic lattice design has been produced, again based on arcs composed of TME cells, joined with long straight sections. The wiggler is distributed between the straights, with injection and extraction on opposite sides of the ring. The beam pipe diameter is $0.064 \mathrm{~m}$, and the dynamic aperture is beyond the physical aperture, up to momentum deviations close to $\pm 1 \%$. The principal parameters are given in Table 4 .

Table 4: Principal Parameters of the Pre-damping Ring

\begin{tabular}{|l|l|l|}
\hline Energy & $E$ & $1.98 \mathrm{GeV}$ \\
\hline Circumference & $C$ & $218.336 \mathrm{~m}$ \\
\hline Number of stored trains & & 2 \\
\hline Natural emittance & $\gamma \varepsilon_{0}$ & $102.7 \mathrm{~mm}-\mathrm{mrad}$ \\
\hline Tunes & $\mathrm{v}_{\mathrm{x}}, \mathrm{v}_{\mathrm{y}}$ & $8.907,7.237$ \\
\hline Natural chromaticity & $\xi_{\mathrm{x}}, \xi_{\mathrm{y}}$ & $-10.39,-12.23$ \\
\hline Momentum compaction & $\alpha$ & $7.05 \times 10^{-3}$ \\
\hline RF voltage/acceptance & $V_{\mathrm{RF}} / \varepsilon_{\mathrm{RF}}$ & $3.40 \mathrm{MV} / 1.5 \%$ \\
\hline Energy spread (rms) & $\sigma_{\delta}$ & $0.092 \%$ \\
\hline Bunch length (rms) & $\sigma_{\mathrm{z}}$ & $7.19 \mathrm{~mm}$ \\
\hline Integrated wiggler field & $\int_{\mathrm{w}_{\mathrm{w}}}{ }^{2} \mathrm{ds}$ & $55.125 \mathrm{~T}{ }^{2} \mathrm{~m}$ \\
\hline Energy loss/turn & $U_{0}+U_{\mathrm{w}}$ & $288+273 \mathrm{keV}$ \\
\hline Damping times & $\tau_{\mathrm{x}, \mathrm{y}, \varepsilon}$ & $4.24,5.13,2.87 \mathrm{~ms}$ \\
\hline
\end{tabular}

Further lattice developments will focus on changes to ease the wiggler design constraints, and lowering the RF voltage. Studies of collective effects are planned.

\section{REFERENCES}

[1] J.N. Corlett et al, "The Next Linear Collider Damping Ring Complex", these proceedings.

[2] J. Sheppard et al, "Update to the Next Linear Collider Injector System Design", these proceedings.

[3] T.O. Raubenheimer and P. Emma, "A Systematic Approach to Damping Ring Design", Phys.Rev.ST Accel.Beams 4:021001, 2001.

[4] R. Rimmer et al, "An RF Cavity for the NLC Damping Rings", these proceedings.

[5] P. Emma and T.O. Raubenheimer, "Circumference Correction Chicanes for Damping Rings", proceedings PAC 1999.

[6] A. Wolski and J.N. Corlett, "Effects of Damping Wigglers on Beam Dynamics in the NLC Damping Rings", these proceedings.

[7] C. Steier, private communication (2001).

[8] J.N. Corlett et al, "Impedance and Instabilities in the NLC Damping Ring", these proceedings.

[9] C. Steier et al, "Intra-Beam Scattering and Minimum Achievable Emittance in the Advanced Light Source", these proceedings. 